\title{
Study on the Intelligence Structure of Current
}

\section{Differential Protection in Transmission Line of UHV Based on Agent}

\author{
Zhenyu Chen \\ Guangdong Business College \\ South China University of Technology, Guangzhou 510320, China \\ Tel: 86-20-38490856_E-mail: chenzy9908@126.com \\ The Project is supported by Guangdong Provincial Natural Science Foundation of China (0602396)
}

\begin{abstract}
At present, action criterions of Phase-segregated current differential protection in Transmission line of UHV include the criterion based on whole current, instantaneous value of fault and other changing from it. In different areas of fault, each criterion has different reliability and sensitivity. On the basis of deeply analyses and study of the principle for Phase-segregated current differential protection, this paper combines intelligence Agent technology, and creates an intelligent protection structure which can choose reasonable action criterion according to the place of fault, and it can make the reliability and sensitivity of Phase-segregated current differential protection in Transmission line of UHV improved.

Keywords: Transmission line of UHV, Phase segregated and differential current Protection, Action criterion, Intelligent Agent

With the rapid development of the global economy, the power demand is more and more growing, large-scale, high-capacity and long-distance power transmission has become a form of modern power system development. But comparing UHV transmission with the traditional HV and EHV transmission, the obvious economic and technical superiority are presented, especially, they are widely applied to attention in foreign and China power system ${ }^{[1]}$. In order to satisfy the development of the Northwest power grid to solve large-capacity power send out from west to east, adopting UHV transmission is a general trend, so the country's first of UHV transmission lines has been built in china: the $750 \mathrm{kv}$ single system about $146 \mathrm{~km}$ from GuanTing to LanZhou East. Compared with other EHV(330kv,500kv,etc), the $750 \mathrm{kv}$ transmission lines UHV brings about greater capacity of current and longer distance transmission, the too high voltage and capacitance current caused by short circuit impact on the System reliability and stability. To achieve this, we should learn from foreign experience in the design of protective relaying UHV transmission lines, fewer detours, and speed up the pace; On the other hand, we must in-depth study line protection and create the basis for the theory for the design and manufacture of UHV line protection, but also to ensure the stability operation of system.
\end{abstract}

\section{Phase-segregated current differential protection}

\subsection{Principles and characteristics of the Phase-segregated current differential protection}

The basic principles: It transferred the time-lable current information and data packets by communication link from the end to the other end; it realizes the balance and phase calculations between two ends. From this, we can judge whether the breakdown will happen or not.

Looking from the principle of protection, Phase-segregated current longitudinal differential protection is an ideal situation; it is not affected by the oscillation, the mode of operation and the resistance ${ }^{[2]}$. Meanwhile, the Phase-segregated differential current protection, without the introduction of voltage avoid a transient voltage secondary circuit complexity in ultra-high voltage power grid, so comparing with the longitudinal direction for the protection and longitudinal distance (zero sequence) protection, Phase-segregated current longitudinal differential protection principles are simple, almost without affecting by the voltage transformer (TV) lost, contact compensate, same tower or many other factors, it has long been considered to be professionals ideal transmission line protection program ${ }^{[3]}$.

Their characteristics are manifested mainly in the following aspects: 
(1) Current differential protection only measured on the current value, and does not calculate the fault distance impedance. The influence of greater arcing resistance to the protection does not exist in the EHV and the UHV system.

(2) The current differential protection makes the measurements for the current balance and phase at both ends, so the fault phase can be elected clearly, and makes the fault phase correct easily.

(3) The current differential protection system is not influenced by system oscillation. When the system is oscillating, the direction of current at the two ends is the same as it is normally operating and they have the identical swings of phase. Even when the oscillation system fault protection device is able to act correctly according to different current phase.

(4) Current differential protection is not affected by voltage and current of opposite phase in the contact compensate system. Because the protection methods only relate with the current numerical and phase at both ends, it does not involve the phase relationship between voltage and current.

In addition, differential current protection is not affected by the load current and the problems in the three-phase short-circuit death zone. Therefore, in approaching UHV transmission line protection, the technology of phase-distance differential protection will replace the high-frequency technology, and become the mainstream technology of UHV transmission line protection ${ }^{[4]}$.

\subsection{The issues which exist in UHV current differential protection}

UHV which carries with a capacity of current for a long-distance owns economic and technical advantages, but also it will face two major problems: First, because of the high voltage circuits and the high insulation costs, if it can promptly lower voltage level after the fault? Second, because the transmission line brings about the larger electric capacity current, the impact is particularly large for protection.

For the first issue, as storage elements exist and its energy cannot mutate, it will exert over -voltage in the UHV fault lines when it puts into empty and breaks the fault. To ensure that the insulator was no breakdown when the over-voltage presents, the program can be taken:

(1) Increasing the level of insulation (but increase investment);

(2) Through the rational allocation and choose arrester to prevent the electrical equipment damage from the over-voltage (but it will generate conduction from lightning arrester to earth, and expand the scope of protection);

(3) Adding shunt induct coil (but it will result the operation complexity between it and breaker)

(4) Set up a reasonable order for protective actions and circuit breaker tripping in order to limit the over-voltage (operating experience of UHV lines in Russian proves that the method is effective at certain extent).

Regarding the second question, the $750 \mathrm{kv}$ transmission line is composed of six divide wire. Comparing with 500kV transmission lines, its wire diameter increases, its impedance declines, and its capacity of transport makes greater. But also the distributed capacitance current increases between different phases and phase to earth, the current both ends of the lines isn't balance in the normal functioning of state, thus it results poor current, and it will become even more noticeable in failure. The problem may be not serious on the general the differential current protection of the HV and EHV long line, but for the UHV transmission line, it may be fatal. So on the EHV of long line, studying current differential protection should be given sufficient attention.

\subsection{Action criterions apply to current differential protection of UHV transmission lines}

After study in $750 \mathrm{kv}$ transmission line, the capacitive current will reach or surpass $100 \% \mathrm{I}_{\mathrm{e}}$ when the best transmission radius is more than $1000 \mathrm{~km}^{[6,7]}$, with the growth of line, capacitive current is also growing. Simulation experiments prove that the flow of capacitive current passing through differential relay can be achieved 1270A in none measures and normal working, and it accounts for $52.88 \%$ of the flow through the current phase. To protect from malfunction, it is necessary to increase the differential fixed threshold value of the protection, but it will reduce the protective sensitive, and make it difficult to set, it can easy lead to protection malfunction ${ }^{[7]}$. Therefore, high voltage shunt reactor are inserted in the actual transmission line to compensate part of capacitive current, reduce the influence and increase sensitivity of capacitive current differential protection, but when the line is longer, the circuit will still be larger capacitive current, it will affect the normal operation of the protection.

Therefore, how to raise the sensitive of protection at the same time, improve the reliability of the protection movement, is the focus for us. At present, it is more effective way to study in the criterion. there are usually two ways of Criterions used on, they are whole current and fault component, as well as Normalization criteria and the instantaneous value of the fault component criteria transforming from these. Their characteristics are following:

(1) The whole current action criterion: 


$$
\begin{aligned}
& \left|\dot{I}_{m}+\dot{I}_{n}\right|>I_{z d} \\
& \left|\dot{I}_{m}+\dot{I}_{n}\right|>K\left|\dot{I}_{m}-\dot{I}_{n}\right|+\dot{I}_{0}
\end{aligned}
$$

if the criterion is Adopted during normal operation or external fault, the existence of load current will not have a huge impact protection, protection reliably can not cut off breaker, but in the case of an internal asymmetric fault or sending out large load by big electric resistance, the protection will doesn't action, and reduce its sensitivity.

(2) The criterion of fault components:

$$
\begin{aligned}
& \left|\Delta \dot{I}_{m}+\Delta \dot{I}_{n}\right|>\Delta \dot{I}_{z d} \\
& \left|\Delta \dot{I}_{m}+\Delta \dot{I}_{n}\right|>K\left|\Delta \dot{I}_{m}-\Delta \dot{I}_{n}\right|
\end{aligned}
$$

The criterion of phase constituted differential protection is maid of mutations current after system failures in each phase current volume, due to adopt a fault component currents, it eliminates the impact of load current from the principle, the capability of transit resistance has been markedly strengthened, and higher sensitivity of the action. However, we need some time to eliminate the DC component, it has not higher speed.

On the basis of criteria similar to the birth:

(3) The criterion of normalization:

$$
\left|\frac{I_{m}}{\left|I_{m}^{\prime}\right|}+\frac{I_{n}}{\left|I_{n}\right|}\right|>K\left|\frac{I_{m}}{|| I_{m}^{\prime} \mid}-\frac{I_{n}}{\left|I_{n}\right|}\right|
$$

By simulation the criterion is applicable to the all conditions for import as fault components, it respond quickly in the region, and has certain the high reliability to external region.

(4) The fault component criteria based on the instantaneous value:

$$
\begin{aligned}
& \sum_{k=t_{0}}^{t_{k}} \Delta i_{m}(k) \bullet \Delta i_{n}(k) \geq E_{d z} \\
& \sum_{k=t_{0}}^{t_{k}}\left|\Delta i_{m}(k)\right| \geq k \sum_{k=t_{0}}^{t_{k}}\left|\Delta i_{n}(k)\right|
\end{aligned}
$$

The criterion maid use of instantaneous value can be avoided as the complex Fourier calculate, and reduce the time of action, at the same time it has the actions properties of against time. The more internal fault is serious, the more the current fault component is greater, and the speed is more faster that the fault was judged, it replenish the insufficiency to the serious fault for failures of rapid response to, the differential protection in internal fault has the best sensitivity.

Different criterion has itself expertise, in order to enhance the protection of UHV, We designed a agent architecture from the perspective of artificial intelligence, the agent architecture can dynamically choose different criteria in different fault region, it can coordinate each other and choose reasonable, it can effectively improve the reliability and sensitivity in the UHV transmission lines.

\section{Intelligent Agent}

"Agent" is also known as act on behalf of someone in a responsible position, in a sense, it has the characteristics of commercial activities with factor, it has the ability of their own reaction, coordination and decision-making according to environmental changes. The technology of Intelligent Agent has become one of the tasks of the fastest-growing in artificial intelligent field, it is a collection of autonomy, social response, adaptability and coordination of integrated, an intelligent entity formed by the hardware or software. Their identity is mainly manifested in the following aspects:

(1) Autonomy: Agent operation is noninterference directly by human or other agent, its implementation is different from ordinary procedures controlled entirely by outside, not as objects, the definition of its internal transfer from the outside, Agent has a certain extent for the state of their internal and the action;

(2) Society: Agent can achieve coordination and interaction with the external environment or other agents through a 
certain language (e.g. KQML);

(3) Response: Agent has the ability of perceiving the environment and responding and impacting on the environment in time;

(4) The nature of adaptation: Agent has the character of opening, it enable to adapt the inference activities gradually , and expand, restrict or amend their own state of local knowledge, keep the target at the right time;

(5) Coordination: with the environment or with other Agent: consultations, cooperation and coordination work.

\section{The individual Agent Architecture applied to differential protection}

According to the features of differential protection, we have built a differential protection Agent with intelligent behavior; it can automatically choose different criteria of differential protection according to the different fault to achieve the greatest sensitivity and reliability. Its structure is as follows:

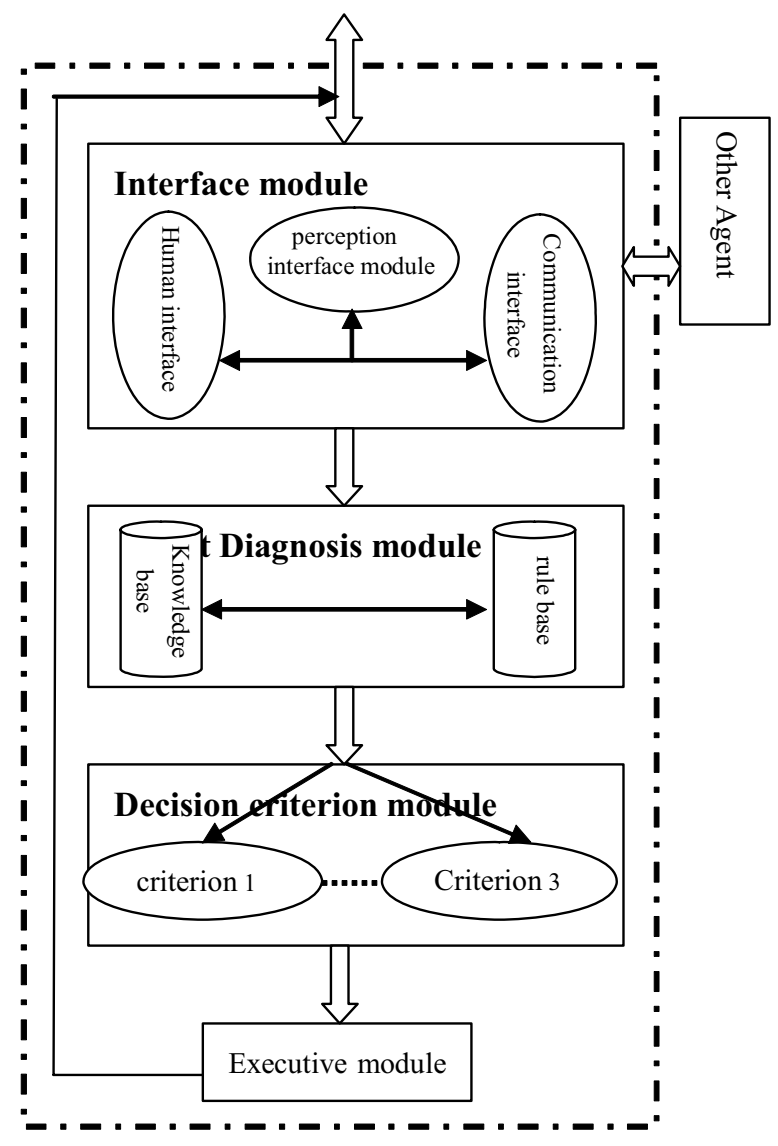

Figure 1. Structure of individual agent

The structure consists of six modules, each module fulfills its task as commitment, here there are some main functions:

(1) Interface Module

Interface Module includes perception interface module, human interface module and communication interface module three parts.

Perception interface module which is the interface between Individual Agent and the surrounding environment, it is used to Percept the changes for the environment around; the man-machine interface module is the interface between Individual Agent and the user interaction, it is used to receive user information or instructions and return to the results of implementation; Communication Interface module is used to exchange the information with other agent, help each other, and accomplish a tasks with cooperate.

(2) Fault diagnosis module

Fault diagnosis module analysis the fault information transmitting by perception interface module for calling the knowledge base and rule base, and find out the cause of the malfunctions, identify faults component and judge fault types. This is very important to select accurate criterion for decision criterion module at the back; 


\section{(3) Decision criterion module}

There are many criterions in the decision criterion module, as: the whole current action criteria, the fault component criteria based on the instantaneous value and the criterion of normalization. They can select correctly relevant criterion through diagnosing different fault type by Fault diagnosis module, (e. g fault in or out), carry out correct action result, the sensibility and the credibility of protect get valid exertive.

(4) Executive module

Executive module carry out the correspond behavior according to the result performance of the decision criterion module.

The protect model makes use of a forerunner artificial intelligence technique research result, feels fault of transmission line passing by perception interface module on the basis of extensive use for fixed fault position ${ }^{[10]}$ and fault diagnosis ${ }^{[11]}$, delivers the possession information that has been diagnosed by fault diagnosis module to decision criterion module, then selects relevant action criterion by Decision criterion module. While examining a fault to break down and may take place in the area, it chooses the fault component criteria based on the instantaneous value (4) as lord protection, so it can raise the sensitivity of protection in the area; While examining a fault to break down and may take place out the area, it chooses the whole current action criteria (1) as lord protection, it may raise the credibility of the action when tremendous exaltation exterior break down. The criterion of normalization (3) is look on as near protection backup for this line, after it is delayed, if lord protection can act credibility, the protection return, otherwise it breaks down this line after certain time, it can achieve the greatest sensitivity and reliability.

\section{Emulate result}

Following fig is a emulate model:



Figure 2. The simulate model in $1000 \mathrm{KV}$ power transmission

The paper has calculated to a power system of $1000 \mathrm{KV}$ by making use of a ATP emulate procession for electromagnetic transient status. Electric source is expressed with concentrate parameter, line is expressed with LCC, and concrete parameter is as follows:

Power: $\quad E M=1000 \angle 0 \quad k V, \quad E_{N}=1000 \angle \delta k V ; \quad Z_{M 1}=0.4225+j 29.85 \Omega, \quad Z_{M 0}=0.1865+j 26.71 \Omega, \quad Z_{N 1}=0.4225+j 29.85 \Omega$, $\mathrm{Z}_{\mathrm{N} 0}=0.1865+\mathrm{j} 26.71 \Omega$.

LCC model using lines: about $50 \mathrm{~m}$ high towers, three-phase horizontal arrange in a row, $22 \mathrm{~m}$ of an average height to earth, $24 \mathrm{~m}$ of the distance between the two phase, $45.5 \mathrm{~m}$ of an altitude on average and $34.6 \mathrm{~m}$ of the distance for two overhead ground wire, protection angle is $24^{\circ} \sim 28^{\circ}$. voltaic wire are maid of eight separatist wire, each of wire diameter is $35 \mathrm{~mm}$ and $400 \mathrm{~mm}$ between them, diameter of the entire split wire is about $1000 \mathrm{~mm}$.

In addition, there is compensation in tandem with the capacitor and Shunt Reactors compensation. The compensation will reach $110 \%$. Total installed capacity of compensation 25800MVAr shunt reactor, 13500MVAr series capacitor.

The simulation results show that: when the fault break down inside the sanctuary at $\mathrm{f}_{1}$ point, the lord protection criterion which is constituted of the instantaneous value and the fault component can rapid cut off breakers at the two ends of lines; When the fault occurred outside the sanctuary at $f_{2}$ point, the lord protection criterion which is constituted of whole current action criteria can refuse to cut off the line. Intelligent Protection realized in different regions of the fault, will automatically, and the dynamic select the different criterion, the sensitivity and reliability of the protection have been effectively raised. But the coordination among different segments and protections on Transmission line will be the contents for next step to study.

\section{References}

[1] Barabanov, Yoriy. (2001). Protection Relays of 750kv Transmission Line in Russia. (He Jiali,Trans In: Report of Academic Workshop of Beijing Society of Electrical Engineering ). Beijing.

[2] Fan, Zhanfeng, Li, Rui-sheng, Zhang Hong-xia and Li Zheng-feng. (2005). Research on UHV transmission line 
protection pilot differential protection. Relay. Vol.33. No.3. February 1.

[3] Zhu, Shengshi. (1995). Principle and Technology of Protective Relaying in HV System. Beijing: China Electric power Press.

[4] Li, Zhe. (2000). Discuss the Application of Split-phase Current-Differential Protection in EHV Transmission Lines. Plan and design.

[5] The Prospect of Developing UHV Transmission System in China. UHV technology. Beijing: 2002.

[6] Dong, Xinzhou, Su, Bin, Bo Zhiqian and He Jiali. (2004). Study of Special Problems on Protective Relaying of Transmission Line. Automation of Electric Power Systems. Vol.28, No.22. Nov. 25. No. 4.

[7] Wang, Haigang, Dong Xinzhou, Bo, Zhiqian. (2006). A Sensitive and Operational Criterion for Current Differential Protection of Transmission Line. Power system technology. Vol. 30. No.10. 5.

[8] Qian, Feng. (2005). The Outline and Application of the New Fault Place Theory and Technology on Line. The Supervise Equipment and Fault Diagnosis in power System. 11.

[9] Nie, Qinwu. (2004). Research on Distributed Intelligence Fault Diagnosis Model \& Implementation Which Based on MAS. Engineering and application of computer. 10

[10] Bo, Z. Q. (2002). Adaptive non-communication protection for power lines BO scheme I - the delayed operation approach. IEEE Trans on Power Delivery, 17(1): 85-91.

[11] Li, Yan, Chen, De-shu, Zhang Zhe, et al. (2001). The emulation analysis for the influence of capacitance current of UHV transmission line on differential current protection and compensating countermeasure. Relay. 29(6):6-9.

[12] Coury, D. V., Thorp, J. S., Hopkinson, K. M. et al. (2002). An Agent-based current differential relay for use with a utility Intranet. IEEE Trans on Power Delivery. 17(1): 47-53. 